\title{
The glycoprotein TRP36 of Ehrlichia sp. UFMG-EV and related cattle pathogen Ehrlichia sp. UFMT-BV evolved from a highly variable clade of $E$. canis under adaptive diversifying selection
}

\author{
Alejandro Cabezas-Cruz ${ }^{1,2^{*}}$, James J Valdés ${ }^{3}$ and José de la Fuente ${ }^{2,4^{*}}$
}

\begin{abstract}
Background: A new species of Ehrlichia, phylogenetically distant from E. ruminantium, was found in 2010 infecting cattle in Canada. In 2012 and 2013, we reported the in vitro propagation, molecular and ultrastructural characterization of Ehrlichia sp. UFMG-EV (E. mineirensis), a new species of Ehrlichia isolated from the haemolymph of Brazilian Rhipicephalus (Boophilus) microplus ticks. A new organism, named Ehrlichia sp. UFMT-BV, closely related to Ehrlichia sp. UFMG-EV, was recently described in Brazil and after experimental infection it was shown to be pathogenic for cattle. This new emerging clade of cattle Ehrlichia pathogens is closely related to E. canis. The major immunogenic Tandem Repeat Protein (TRP36; also known as gp36) is extensively used to characterize the genetic diversity of $E$. canis. Homologs of TRP36 were found in both Ehrlichia sp. UFMG-EV and Ehrlichia sp. UFMT-BV.
\end{abstract}

Findings: Herein, we characterized the evolution of this new Ehrlichia clade using TRP36 sequences. Our working hypothesis is that Ehrlichia sp. UFMG-EV and related microorganisms evolved from a highly variable E. canis clade. In support of our hypothesis we found that Ehrlichia sp. UFMG-EV and Ehrlichia sp. UFMT-BV TRP36 evolved from a highly divergent and variable clade within E. canis and this clade evolved under episodic diversifying selection with a high proportion of sites under positive selection.

Conclusion: Our results suggest that Ehrlichia sp. UFMG-EV and Ehrlichia sp. UFMT-BV evolved from a variable clade within E. canis.

Keywords: Ehrlichia sp. UFMG-EV, Ehrlichia sp. UFMT-BV, E. mineirensis, Host-shift, Diversifying episodic selection

\section{Findings}

Ehrlichia sp. UFMG-EV and Ehrlichia sp. UFMT-BV belong to a new clade of cattle-related Ehrlichia

Anaplasmataceae is a family of $\alpha$-proteobacteria that includes the genera Anaplasma, Ehrlichia, Neorickettsia and Wolbachia. From these genera, Ehrlichia and Anaplasma are important pathogens affecting animals and humans. Ehrlichia are obligate intracellular gram-negative, tickborne bacteria that grow within membrane-bound vacuoles in human and animal leukocytes causing ehrlichiosis. With

\footnotetext{
* Correspondence: cabezasalejandrocruz@gmail.com; jose_delafuente@yahoo.com ${ }^{1}$ Center for Infection and Immunity of Lille (CIIL), INSERM U1019 - CNRS UMR 8204, Université Lille Nord de France, Institut Pasteur de Lille, Lille, France ${ }^{2} \mathrm{SaBio}$, Instituto de Investigación de Recursos Cinegéticos IREC-CSIC-UCLM-JCCM, Ronda de Toledo s/n, 13005 Ciudad Real, Spain Full list of author information is available at the end of the article
}

a worldwide distribution ehrlichioses are considered emerging diseases that can cause serious illness in a variety of hosts, including humans, livestock and pets. Three news species of cattle-related Ehrlichia spp have been recently reported: (i) a new species that naturally infect cattle from British Columbia, Canada [1], (ii) Ehrlichia sp. UFMG-EV (referred as E. mineirensis in $[2,3]$ ) that was isolated from R. microplus hemolymph [2-4], and (iii) Ehrlichia sp. UFMT-BV that was found to be pathogenic for cattle in Brazil [5]. These three organisms are closely related to E. canis [1,2,5]. Ehrlichia sp. UFMG-EV and Ehrlichia sp. UFMT-BV, however, present new sequence of tandem repeats different to the one reported for E. canis TRP36 [2,5,6]. 
The results of this work expand on our previous findings regarding the evolution and differentiation of TRP36 in Ehrlichia sp. UFMG-EV [2]. Herein, we showed that the gene trp36 presents episodic bursts of selection, unequally distributed across sites and that diversifying selection occurs only in few branches of the trp36 phylogenetic tree. Our results showed that Ehrlichia sp. UFMG-EV and the new Ehrlichia sp. UFMT-BV affecting cattle evolved from a highly divergent and variable clade within E. canis.

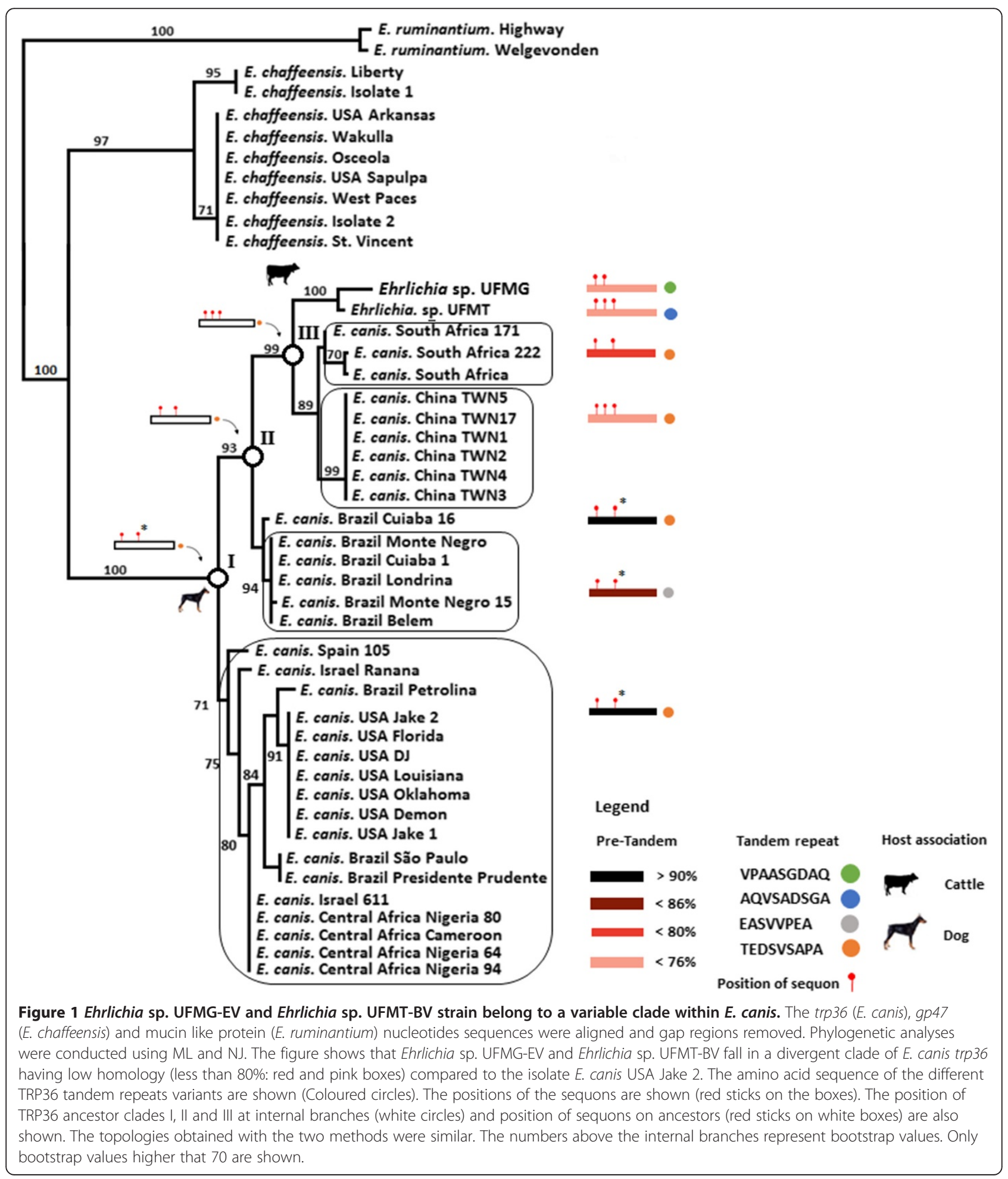


Ehrlichia sp. UFMG-EV trp36 gene evolved from a highly divergent clade within $E$. canis

To study the evolution of trp36 gene we used a combination of phylogenetic and evolutionary analysis (see Additional file 1 for detailed description of materials and methods). The gene trp36 has been widely used to study the genetic diversity of E. canis strains [7-10]. We performed maximum likelihood and neighbor joining phylogenetic analyses with trp36 nucleotide sequences available in GenBank (Additional file 1) to study the evolution of Ehrlichia sp. UFMG-EV and Ehrlichia sp. UFMT-BV trp36 in relation to E. canis trp36. The phylogenetic analysis showed that Ehrlichia sp. UFMG-EV and Ehrlichia sp. UFMT-BV trp36 are separated but clustered together with E. canis strains from South Africa, Taiwan and Brazil (Figure 1). Using the E. canis strain USA Jake-2 as a reference, the TRP36 amino acid sequences from the Taiwanese and South African E. canis strains, together with Ehrlichia sp. UFMG-EV and Ehrlichia sp. UFMT-BV, presented the lowest percent $(<86 \%)$ of homology (Figure 1, red and pink boxes). The results demonstrated that $E$. canis strain USA Jake-2 belongs to a conservative TRP36 clade within E. canis (Figure 1). Members of this clade have a high percent $(>90 \%)$ of amino acid homology in TRP36 (Figure 1, black boxes).

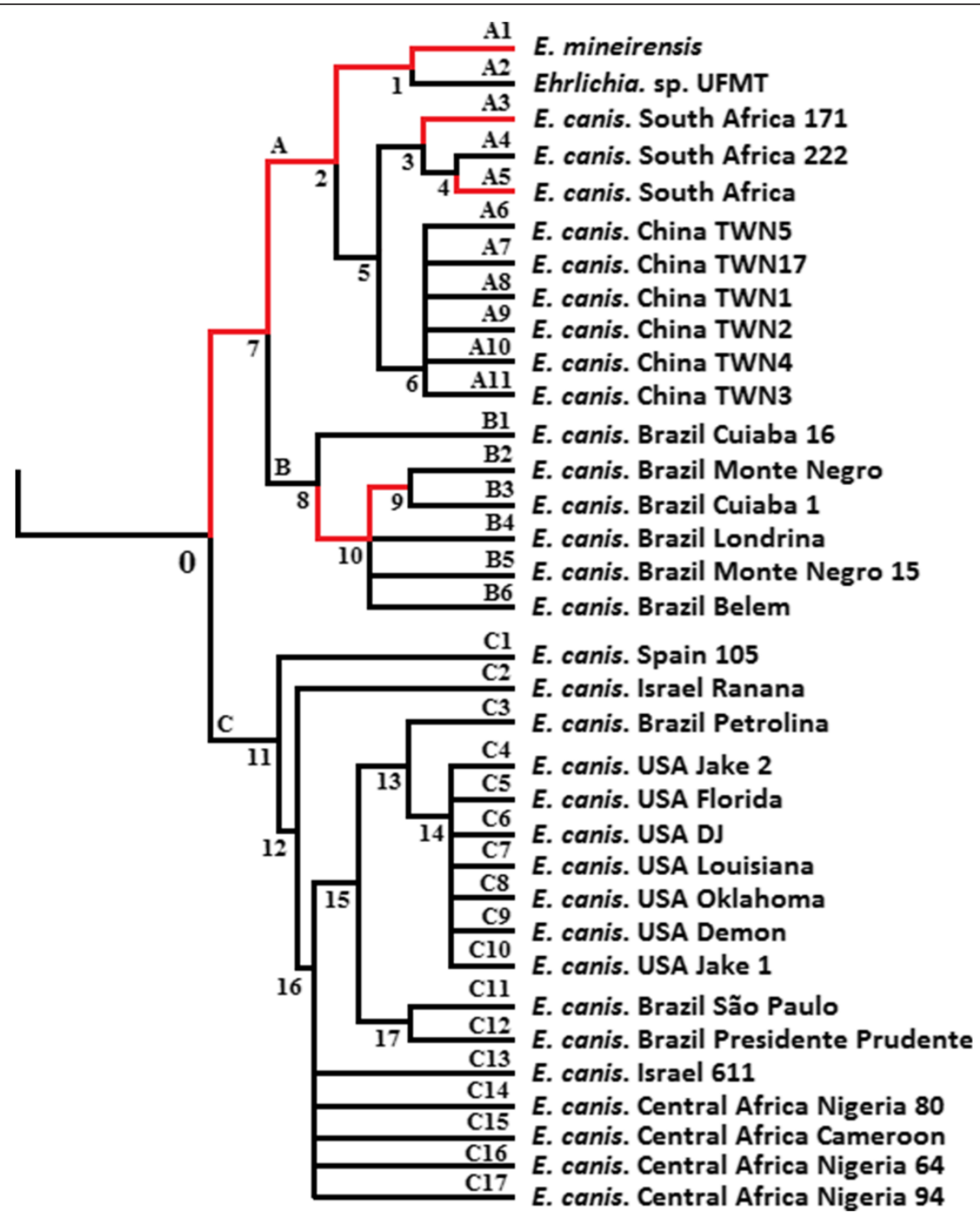

Figure 2 Branches under episodic diversifying selection in the trp36 tree. The tree of trp36 orthologs is shown. Branch-site REL model (Additional file 1) was used to determine branches under episodic diversifying selection (highlighted in red). Branches were considered under episodic diversifying selection when corrected $p$-value $<0.05$ (see Additional file 1 for methods). For the rest of the tree (branches in black) there is no evidence of episodic diversifying selection (Additional file 2). 
The new TRP36 tandem repeat variants evolved from the typical $E$. canis tandem repeat

The tandem repeat composition of the divergent clade was highly variable, encoding the typical $E$. canis TRP36 tandem repeat (TEDSVSAPA), but also other variants AQVSADSGA (Ehrlichia sp. UFMT-BV), EASVVPEA (New Brazilian variant of E. canis) and VPAASGDAQ (Ehrlichia sp. UFMG-EV) (Figure 1, coloured circles). The conservative TRP36 clade, however, only presented the tandem repeat variant TEDSVSAPA amongst all members. Ancestral sequence reconstruction (see Additional file 1 for detailed description of ancestral sequence reconstruction methods) showed that all the new TRP36 variants evolved from the typical TRP36 tandem repeat, TEDSVSAPA (Figure 1, white circles and roman numerals).

There is currently no experimental evidence that TRP36 has N-linked glycans. The evolution of highly divergent variants of TRP36, however, was associated with an increase in the number of sequons of $\mathrm{N}$-glycosylation in TRP36 (Figure 1, red sticks on colored boxes). In agreement with this finding, the evolution of TRP36 ancestors from clades I to III was associated with the gain of one sequon of $\mathrm{N}$-glycosylation for each evolutionary step (from I to II and from II to III - Figure 1, red sticks on white boxes). One of three sequons present in the ancestor of TRP36 clade III was lost in Ehrlichia sp. UFMGEV and in the South African strains, but it is present in Ehrlichia sp. UFMT-BV and the Taiwanese strains. The second sequon in TRP36 ancestor clade I and the strains from USA, Spain, Israel, Central Africa and Brazil possess a proline $(\mathrm{P})$ residue in the second position making it improbable that the asparagine $(\mathrm{N})$ will be glycosylated (Figure 1, asterisks on red sticks). The relevancy of whether these sequons are glycosylated or not is that changes in glycosylation patterns may contribute to evade host immune system [11] and antigenic drift [12].

\section{Ehrlichia sp. UFMG-EV trp36 evolved under episodic diversifying selection}

Our next step was to test whether different branches or codon sites of the trp36 phylogenetic tree evolved under episodic diversifying selection. Results showed that the diversifying selection events among the branches were scarce along the phylogenetic tree (Figure 2). Only 8 (A1, A3, A5, 1, 2, 7, 9 and 10) out of 51 (15.6\%) branches were found to be under episodic diversifying selection (Corrected $p$-value $\leq 0.05$ - Figure 2 and Additional file 2). Episodic diversifying selection was detected only in branches belonging to the highly divergent clade of TRP36 described above (Figure 1). The patterns of episodic diversifying selection were complex, with differences in extent and strength of selection along the diversifying branches. The branches can be separated into four groups: (i) 2, 9, A1, A3 and A5 that experienced strong selective force $(\omega+>3333.56)$ in a small proportion of sites (Proportion $<0.07$ ), (ii) 1 that experienced low selective force $(\omega+=7.86)$ in a high proportion of sites (Proportion $=0.17$ ), (iii) 7 that experienced low selective force $(\omega+=46.08)$ in a low proportion of sites (Proportion $=0.05)$, and (iv) 10 that experienced middle selective force $(\omega+=166.14)$ in a high proportion of sites (Proportion $=0.15)$. Among the branches experiencing episodic selection, 11 out of 171 (6.4\%) codon sites were under episodic diversifying selection (Table 1, Additional file 3). Most of these sites were concentrated in branches 7 and 1 .

Searching the sequences for evidence of positive and negative selection using SLAC, FEL, REL and MEME (see materials and methods) showed that many sites experienced positive or negative selection (Table 2). The higher proportion of sites inferred to be evolving under positive selection was found in the ancestral branches 1 , 7 and 10. The branches A1, A2, B2-B6, which are associated to deep branches 1 and 10 (Figure 2), were related to the occurrence of new forms of TRP36 tandem repeats (Figure 1). This relation suggests that early, strong selective events on lineages 1 and 10 may have been related to the occurrence of new tandem repeats. The sites under negative selection were concentrated in ancestral lineage 2 .

Codon 77 evolved under diversifying (positive) and codon 116 evolved under negative selection. These two codons code for amino acids involved in the formation of sequons among TRP36 homologs (Additional file 4).

\begin{tabular}{|c|c|c|c|c|}
\hline Codons & Branches $^{a}$ & From & To & Type of substitution $^{b}$ \\
\hline 10 & 7 & $\operatorname{aac}(\mathrm{N})$ & ggt $(G)$ & $\mathrm{dN}$ and $\mathrm{dS}$ \\
\hline 21 & 7 & саa $(\mathrm{Q})$ & tca $(S)$ & $\mathrm{dN}$ \\
\hline \multirow[t]{2}{*}{28} & 7 & tca $(S)$ & gta $(V)$ & $\mathrm{dN}$ \\
\hline & 1 & gta $(V)$ & aca (T) & $\mathrm{dN}$ \\
\hline \multirow[t]{2}{*}{39} & 7 & cat $(H)$ & agt $(S)$ & $d N$ \\
\hline & 1 & agt $(S)$ & cat $(\mathrm{H})$ & $\mathrm{dN}$ \\
\hline 40 & 7 & $\operatorname{cct}(P)$ & ggt $(G)$ & $d N$ \\
\hline 51 & 6 & aat $(\mathrm{N})$ & ggt $(G)$ & $d N$ \\
\hline \multirow[t]{2}{*}{77} & 15 & gct $(A)$ & gtt $(V)$ & $d N$ \\
\hline & B1 & gct $(A)$ & gtt $(V)$ & $d N$ \\
\hline 105 & 2 & tat $(Y)$ & gaa (E) & $d N$ \\
\hline 124 & 5 & aat $(\mathrm{N})$ & tct $(S)$ & $\mathrm{dN}$ \\
\hline \multirow[t]{2}{*}{142} & 8 & $\operatorname{tct}(S)$ & ggt $(G)$ & $\mathrm{dN}$ \\
\hline & 10 & tct $(S)$ & gaa (E) & $\mathrm{dN}$ and $\mathrm{dS}$ \\
\hline \multirow[t]{2}{*}{145} & 1 & gct $(A)$ & gtt $(V)$ & $d N$ \\
\hline & $\mathrm{A} 1$ & gtt $(V)$ & caa $(Q)$ & $\mathrm{dN}$ and $\mathrm{dS}$ \\
\hline
\end{tabular}

anternal and external branches are identified by numbers and letters and numbers as in Figure 2.

${ }^{\text {b}}$ Type of substitution: non-synonymous (dN) and synonymous (dS). 
Table 2 Codons under positive and negative selection

\begin{tabular}{|c|c|c|c|c|c|c|c|c|c|c|}
\hline Codons & Branches $^{\mathrm{a}}$ & $\operatorname{SLAC} \omega^{\mathbf{b}}$ & SLAC p-value & FEL $\omega^{\mathbf{b}}$ & FEL p-value & REL $\omega^{b}$ & Bayes factor & MEME $\omega^{\mathbf{b}}$ & MEME p-value & Selection type ${ }^{c}$ \\
\hline 6 & B5 & -4.047 & 0.333 & -3.159 & 0.081 & -1.126 & 229.790 & - & - & Negative \\
\hline 8 & 7 & -4.604 & 0.293 & -4.597 & 0.071 & -1.096 & 92.479 & - & - & Negative \\
\hline 10 & 7 & -0.686 & 0.787 & -2.465 & 0.583 & -0.149 & 0.595 & $>100$ & 0.030 & Positive \\
\hline 21 & 7 & 4.991 & 0.423 & 3.013 & 0.222 & 0.810 & 3.221 & $>100$ & 0.019 & Positive \\
\hline \multirow[t]{2}{*}{28} & 7 & 8.543 & 0.184 & 5.818 & 0.082 & 1.678 & 28.536 & $>100$ & 0.010 & Positive \\
\hline & 1 & & & & & & & & & \\
\hline \multirow[t]{2}{*}{39} & 7 & 6.912 & 0.371 & 3.907 & 0.444 & 1.265 & 8.352 & $>100$ & 0.013 & Positive \\
\hline & 1 & & & & & & & & & \\
\hline 40 & 7 & 4.047 & 0.444 & 2.432 & 0.350 & 0.476 & 1.985 & $>100$ & 0.011 & Positive \\
\hline \multirow[t]{2}{*}{49} & 1 & -16.858 & 0.031 & -10.024 & 0.007 & -1.120 & 178.481 & - & - & Negative \\
\hline & 6 & & & & & & & & & \\
\hline 51 & 6 & 3.473 & 0.603 & 1.923 & 0.586 & 0.096 & 1.136 & $>100$ & 0.028 & Positive \\
\hline \multirow[t]{2}{*}{52} & 1 & -8.094 & 0.111 & -3.234 & 0.062 & -1.087 & 77.050 & - & - & Negative \\
\hline & 6 & & & & & & & & & \\
\hline \multirow[t]{2}{*}{71} & 1 & -18.415 & 0.026 & -14.187 & 0.004 & -1.121 & 187.132 & - & - & Negative \\
\hline & 2 & & & & & & & & & \\
\hline \multirow[t]{2}{*}{77} & B1 & 4.047 & 0.444 & 2.150 & 0.377 & 0.340 & 1.681 & $>100$ & 0.025 & Positive \\
\hline & 15 & & & & & & & & & \\
\hline 83 & 5 & -4.047 & 0.333 & -3.402 & 0.073 & -1.127 & 243.194 & - & - & Negative \\
\hline 89 & 2 & -9.551 & 0.153 & -7.402 & 0.042 & -1.101 & 99.411 & - & - & Negative \\
\hline 94 & 2 & -6.708 & 0.201 & -4.324 & 0.059 & -1.124 & 189.451 & - & - & Negative \\
\hline 96 & 2 & -4.047 & 0.333 & -4.119 & 0.063 & -1.127 & 242.661 & - & - & Negative \\
\hline 105 & 2 & 3.569 & 0.689 & 2.357 & 0.608 & 0.330 & 1.536 & $>100$ & 0.030 & Positive \\
\hline 116 & 2 & -7.488 & 0.180 & -3.380 & 0.074 & -1.126 & 199.960 & - & - & Negative \\
\hline 124 & 5 & 3.663 & 0.543 & 2.278 & 0.488 & 0.329 & 1.569 & $>100$ & 0.004 & Positive \\
\hline 139 & 10 & -17.080 & 0.037 & -7.190 & 0.074 & -1.007 & 23.196 & - & - & Negative \\
\hline \multirow[t]{2}{*}{142} & 1 & 6.862 & 0.316 & 6.098 & 0.511 & 1.459 & 31.512 & $>100$ & 0.009 & Positive \\
\hline & 10 & & & & & & & & & \\
\hline \multirow[t]{2}{*}{145} & 1 & 4.902 & 0.413 & 4.701 & 0.571 & 1.230 & 7.496 & $>100$ & 0.017 & Positive \\
\hline & $\mathrm{A} 1$ & & & & & & & & & \\
\hline \multirow[t]{3}{*}{152} & 10 & 20.127 & 0.244 & 41.463 & 0.238 & 1.510 & 66.006 & $>100$ & 0.224 & Positive \\
\hline & B4 & & & & & & & & & \\
\hline & A5 & & & & & & & & & \\
\hline \multirow[t]{5}{*}{158} & 1 & 2.355 & 0.585 & -1.195 & 0.886 & 1.162 & 161.941 & 1.219 & 0.612 & Positive \\
\hline & 4 & & & & & & & & & \\
\hline & A4 & & & & & & & & & \\
\hline & A5 & & & & & & & & & \\
\hline & 10 & & & & & & & & & \\
\hline \multirow[t]{4}{*}{162} & 1 & 16.368 & 0.227 & 8.312 & 0.354 & 1.574 & 87.208 & $>100$ & 0.343 & Positive \\
\hline & 5 & & & & & & & & & \\
\hline & 10 & & & & & & & & & \\
\hline & B2 & & & & & & & & & \\
\hline
\end{tabular}


Table 2 Codons under positive and negative selection (Continued)

\begin{tabular}{|c|c|c|c|c|c|c|c|c|c|c|}
\hline \multirow[t]{3}{*}{166} & 1 & 25.338 & 0.042 & 5.483 & 0.239 & 1.510 & 20.855 & $>100$ & 0.198 & Positive \\
\hline & 10 & & & & & & & & & \\
\hline & A8 & & & & & & & & & \\
\hline \multirow[t]{2}{*}{167} & 10 & 28.242 & 0.032 & 8.115 & 0.083 & 1.677 & 53.176 & $>100$ & 0.084 & Positive \\
\hline & B1 & & & & & & & & & \\
\hline \multirow[t]{2}{*}{170} & 10 & -8.379 & 0.406 & -74.785 & 0.031 & 0.536 & 0.396 & - & - & Negative \\
\hline & 9 & & & & & & & & & \\
\hline
\end{tabular}

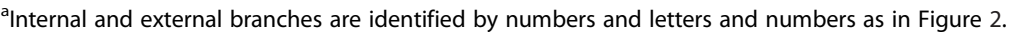

${ }^{b}$ The ratio between non-synonymous (dN) and synonymous (dS) nucleotide substitution per site ( $\boldsymbol{\omega}$ ) analyzed by Datamonkey via SLAC, FEL, REL and MEME.

${ }^{c}$ Sites were considered under positive selection $(\boldsymbol{\omega}>1)$ or negative selection $(\boldsymbol{\omega}<1)$ when at least one of the methods shows significant difference $(p$-value $<0.05$ (SLAC, FEL and MEME) or Bayes Factor $>50$ (REL)).

While codon 77 was selected in branches 15 and B1 (E. canis), codon 116 was selected in branch 2 (E. canis, Ehrlichia sp. UFMT-BV and Ehrlichia sp. UFMG-EV). This data therefore suggests that putative N-glycosylation associated with this sequon might be important in the host shift (see below) observed in Ehrlichia sp. UFMT-BV and Ehrlichia sp. UFMG-EV.

\section{Model of emergence of Ehrlichia sp. UFMG-EV and Ehrlichia sp. UFMT-BV within E. canis}

The emergence of new pathogens is frequently associated to mutations that confer the ability to infect novel hosts, known as "host shift" [13]. Ehrlichia sp. UFMG-EV and Ehrlichia sp. UFMT-BV are closely related to E. canis, however they were associated to new invertebrate and vertebrate hosts, respectively. First, while the common tick vector for E. canis is R. sanguineus [14], Ehrlichia sp. UFMG-EV was isolated from $R$. microplus hemolymph [2]. Secondly, while E. canis is mainly pathogenic for dog [10], Ehrlichia sp. UFMT-BV was found to be pathogenic for cattle [5]. How pathogens can colonize new hosts is a challenging question in evolutionary biology [13]. Recently, Aguiar and colleagues [9] suggested that E. canis may have a wider range of hosts in Brazil than currently recognized. The host shift in this context may have occurred in a scenario where dogs infected with a variable E. canis strain, as previously found in Brazil [9], were the source of infection for $R$. microplus or $R$. sanguineus ticks that later infested cattle. Both tick species are able to infect dogs $[15,16]$ and cattle [17]. The scenario involving $R$. microplus is unlikely as this is a onehost tick species. However, $R$. microplus moves among hosts during their parasitic lifetime [18], thereby increasing the chances of horizontal pathogen transmission among different hosts. Changes in evolutionary pressures on E. canis, related to new host association, may have resulted in a completely new species.

Our evidence supports the idea of differential evolutionary pressures on the glycoprotein TRP36 along different strains of E. canis, resulting in highly divergent variants of
TRP36. In the habitual host of E. canis, TRP36 must possess amino acid positions beneficial or neutral that may be deleterious in new hosts - the opposite may also be true. Within variable strains of a given pathogen, novel genetic variants may eventually deliver beneficial mutations that promote successful emergence, thereby providing a source for adaptive genetic variation in new hosts [13]. In agreement with this, we found a large proportion of sites that evolved under purifying (negative) selection, positive and diversifying selection. It is worth noting that the selective events were more frequent and strong in the deepest branches of trp36 phylogenetic tree. This supports the hypothesis that most mutations that originated in the new TRP36 amino acid variants of Ehrlichia sp. UFMG-EV and Ehrlichia sp. UFMT-BV occurred before the emergence of the clade formed by these two organisms. The fact that the most recent common ancestor (Figure 1, ancestor clade III) between Ehrlichia sp. UFMG-EV, Ehrlichia sp. UFMT-BV and E. canis had a typical TRP36 tandem repeat structure, supports the aforementioned hypothesis. The divergence found in TRP36 tandem repeats was consistent with a $1.7 \%$ sequence divergence between $16 \operatorname{Sr} R A$ of Ehrlichia sp. UFMG-EV and E. canis [2]. Taking into account the high identity of $16 \operatorname{SrRNA}$ among E. canis strains (maximum 0.6\%) [7], and thus the conservative nature of this gene, Ehrlichia sp. UFMG-EV may have diverged a long time ago from E. canis.

\section{Conclusion}

Altogether, these results suggest that this new group of organisms evolved from E. canis sensu stricto and has become ecologically independent from the parental species. In agreement with the new hosts association of this group of microorganisms, it was found that Ehrlichia sp. UFMG-EV was able to propagate in bovine aorta BA886 cell line, while E. canis did not [4]. This in vitro observation supports the above conclusions regarding the new host specificity of this novel group of cattle related agents. At the ultrastructural level, Ehrlichia sp. UFMGEV shares ultrastructural features with other members of 
the genus Ehrlichia (E. muris, E. canis and E. chaffeensis). We found cells, however, with unusual structures (invagination of the cellular membrane) for which we yet do not have an explanation [3]. Further studies should clarify the role of major immunogenic surface exposed proteins in the evolution of bacterial host shift. The full genome of E. mineirensis (Ehrlichia sp. UFMG-EV) might be an important contribution to these studies.

\section{Additional files}

Additional file 1: Detailed description of materials and methods.

Additional file 2: Likelihood ratio test statistics for branches under episodic diversifying selection. Branch-site REL model (Additional file 1) was used to determine branches under episodic diversifying selection (highlighted in red). Branches were considered under episodic diversifying selection when corrected $p$-value $<0.05$ (see Additional file 1 for methods)

Additional file 3: Likelihood ratio test statistics for sites under episodic diversifying selection. MEME (Additional file 1) was used to determine sites (codons) under episodic diversifying selection (highlighted in red). Sites were considered under episodic diversifying selection when $p$-value $<0.05$ (see Additional file 1 for methods).

Additional file 4: Sequons of $\mathrm{N}$-glycosylation in the $\mathrm{N}$-terminus of TRP36 variants. Putative sequons of $\mathrm{N}$-glycosilation of the $\mathrm{N}$-terminus of TRP36 variant included in this study are shown.

\section{Competing interests}

The authors declare that they have no competing interests.

\section{Authors' contributions}

ACC developed the overall concept, drafted the manuscript, performed and interpreted the phylogenetic and evolutionary analysis. JJV drafted the manuscript and performed and helped in the interpretation of phylogenetic and evolutionary analysis. JF drafted the manuscript and made critical revisions to the manuscript and interpretation of the data. All authors read and approved the final version of the manuscript

\section{Acknowledgments}

This research was partially supported by the EU FP7 ANTIGONE project number 278976. JJV was sponsored by project CZ.1.07/2.3.00/30.0032, co-financed by the European Social Fund and the state budget of the Czech Republic. ACC was supported by a grant from the Ministère de l'Education Supérieure et de la Recherche of France.

\section{Author details}

Center for Infection and Immunity of Lille (CIIL), INSERM U1019 - CNRS UMR 8204, Université Lille Nord de France, Institut Pasteur de Lille, Lille, France. ${ }^{2} \mathrm{SaBio}$ Instituto de Investigación de Recursos Cinegéticos IREC-CSIC-UCLM-JCCM, Ronda de Toledo s/n, 13005 Ciudad Real, Spain. ${ }^{3}$ Institute of Parasitology, Biology Centre of the Academy of Sciences of the Czech Republic, České Budějovice, Czech Republic. ${ }^{4}$ Department of Veterinary Pathobiology, Center for Veterinary Health Sciences, Oklahoma State University, Stillwatert, OK 74078, USA.

Received: 24 September 2014 Accepted: 30 November 2014 Published online: 10 December 2014

\section{References}

1. Gajadhar AA, Lobanov V, Scandrett WB, Campbell J, Al-Adhami B: A novel Ehrlichia genotype detected in naturally infected cattle in North America. Vet Parasitol 2010, 173:324-329.

2. Cruz A, Zweygarth E, Ribeiro M, da Silveira J, de la Fuente J, Grubhoffer L, Valdés J, Passos LMF: New species of Ehrlichia isolated from Rhipicephalus (Boophilus) microplus shows an ortholog of the E. canis major immunogenic glycoprotein gp36 with a new sequence of tandem repeats. Parasit Vectors 2012, 5:291.
3. Cabezas-Cruz A, Vancová M, Zweygarthb E, Ribeiro MFB, Grubhoffer L, Passos LMF: Ultrastructure of Ehrlichia mineirensis, a new member of the Ehrlichia genus. Vet Microbiol 2013, 167:455-458

4. Zweygarth E, Schöl H, Lis K, Cabezas-Cruz A, Thiel C, Silaghi C, Ribeiro MFB, Passos LMF: In vitro culture of a novel genotype of Ehrlichia sp. from Brazil. Transbound Emerg Dis 2013, 60:86-92.

5. Aguiar DM, Ziliani TF, Zhang X, Melo AL, Braga IA, Witter R, Freitas LC, Rondelli AL, Luis MA, Sorte EC, Jaune FW, Santarém VA, Horta MC, Pescador CA, Colodel EM, Soares HS, Pacheco RC, Onuma SS, Labruna MB, McBride JW: A novel Ehrlichia genotype strain distinguished by the TRP36 gene naturally infects cattle in Brazil and causes clinical manifestations associated with ehrlichiosis. Ticks Tick Borne Dis 2014, 5:537-544.

6. Doyle CK, Nethery KA, Popov VL, McBride JW: Differentially expressed and secreted major immunoreactive protein orthologs of Ehrlichia canis and E. chaffeensis elicit early antibody responses to epitopes on glycosylated tandem repeats. Infect Immun 2006, 74:711-720.

7. Hsieh YC, Lee CC, Tsang CL, Chung YT: Detection and characterization of four novel genotypes of Ehrlichia canis from dogs. Vet Microbiol 2010, 146:70-75.

8. Kamani J, Lee CC, Haruna AM, Chung PJ, Weka PR, Chung YT: First detection and molecular characterization of Ehrlichia canis from dogs in Nigeria. Res Vet Sci 2013, 94:27-32.

9. Aguiar DM, Zhang X, Melo ALT, Pacheco TA, Meneses AMC, Zanutto MS, Horta MC, Santarém VA, Camargo LMA, McBride JW, Labruna MB: Genetic diversity of Ehrlichia canis in Brazil. Vet Microbiol 2013, 164:315-321.

10. Zweygarth E, Cabezas-Cruz A, Josemans Al, Oosthuizen MC, Matjila PT, Lis K, Broniszewska M, Schöl H, Ferrolho J, Grubhoffer L, Passos LMF: In vitro culture and structural differences in the major immunoreactive protein gp36 of geographically distant Ehrlichia canis isolates. Ticks Tick Borne Dis 2014, 5:423-431.

11. Kobayashi Y, Suzuki Y: Evidence for N-glycan shielding of antigenic sites during evolution of human influenza A virus hemagglutinin. J Virol 2012, 86:3446-3451.

12. Das SR, Puigbò P, Hensley SE, Hurt DE, Bennink JR, Yewdell JW: Glycosylation focuses sequence variation in the influenza $A$ virus $\mathrm{H} 1$ hemagglutinin globular domain. PLOS Pathog 2010, 6:e1001211.

13. Dennehy JJ, Friedenberg NA, McBride RC, Holt RD, Turner PE: Experimental evidence that source genetic variation drives pathogen emergence. Proc Biol Sci 2010, 277:3113-3121.

14. Bremer WG, Schaefer JJ, Wagner ER, Ewing SA, Rikihisa Y, Needham GR, Jittapalapong S, Moore DL, Stich RW: Transstadial and intrastadial experimental transmission of Ehrlichia canis by male Rhipicephalus sanguineus. Vet Parasitol 2005, 131:95-105.

15. Dantas-Torres F: Ticks on domestic animals in Pernambuco, Northeastern Brazil. Rev Bras Parasitol Vet 2009, 18:22-28.

16. Szabó MP, de Souza LG, Olegário MM, Ferreira FA, de Albuquerque Pajuaba Neto A: Ticks (Acari: Ixodidae) on dogs from Uberlândia, Minas Gerais, Brazil. Transbound Emerg Dis 2010, 57:72-74.

17. Mirzaei M, Khedri J: Ixodidae ticks in cattle and sheep in Sistan and Baluchestan Province (Iran). Vet Ital 2014, 50:65-68.

18. Chevillon C, Koffi BB, Barré N, Durand P, Arnathau C, de Meeûs T: Direct and indirect inferences on parasite mating and gene transmission patterns. Pangamy in the cattle tick Rhipicephalus (Boophilus) microplus. Infect Genet Evol 2007, 7:298-304.

doi:10.1186/s13071-014-0584-5

Cite this article as: Cabezas-Cruz et al:: The glycoprotein TRP36 of Ehrlichia sp. UFMG-EV and related cattle pathogen Ehrlichia sp. UFMT-BV evolved from a highly variable clade of $E$. canis under adaptive diversifying selection. Parasites \& Vectors 2014 7:584 